Proc. of the 13th Symposium of Magnetic Measurements and Modeling SMMM'2018, Wieliczka, Poland, October 8-10, 2018

\title{
Interpretation of Loss Separation with the Haller-Kramer Model
}

\author{
M.F. DE CAMPOS \\ UFF - Federal Fluminense University, Av. dos Trabalhadores 420, 27255-125 Volta Redonda RJ, Brazil \\ The Haller-Kramer model for domain structure is reviewed in detail. Magnetic domains and domain walls \\ can be interpreted as the Prigogine dissipative structures. As consequence, processes of reversal of magnetization \\ in a hysteresis cycle can be modeled with the minimum energy production principle. The Haller-Kramer model \\ gives physical basis for the loss separation procedure.
}

DOI: 10.12693/APhysPolA.136.705

PACS/topics: 75.60.Ej, 75.60.Ch, 75.60.-d

\section{Introduction}

The loss separation model has been widely used since at least 1936 [1]. With the aim of clarifying loss separation, the Haller-Kramer model $[2,3]$ will be discussed in detail. The minimum energy production principle of Prigogine [4] is the basis of the Haller-Kramer model $[2,3]$.

Dissipative structures may happen when minimum energy production takes place. The classical example of dissipative structure is given by the Belousov-Zhabotinsky reaction [5]. Another typical example of dissipative structure in materials science is that composed by cells and dislocation cells $[6,7]$. It is noteworthy that the elastic stored energy at the dislocation cells is much larger than inside the cells. Domains and domain walls is an analogous case, with very large energy inside the tiny volume occupied by domain walls.

The formation of domain walls occurs because this reduces the global energy of the system. The magnetostatic energy is significantly reduced with drastic increase of exchange energy, usually evaluated as the domain wall energy $(\gamma)$. As it will be discussed here, this in fact can be interpreted as a Prigogine dissipative structure, and is implicit inside the Haller-Kramer theoretical treatment $[2,3]$.

When the system is in quasi-stationary state, the rate of entropy production is minimum. This is the Prigogine minimum energy production principle [4]. The Gibbs free energy is given by $G=H-T S$, where $H$ is enthalpy, $T$ is temperature, and $G$ is Gibbs free energy. For constant temperature, and since the enthalpy does not vary with time, $\mathrm{d} G / \mathrm{d} t=0$ also can be considered for expressions representing the minimum energy production.

e-mail: marcosflavio@id.uff.br

\section{The Haller-Kramer model and the loss separation}

The Haller-Kramer model takes into account the total energy of the system, including domain walls (exchange energy), magnetostatic energy, and eddy currents. As the global energy of the system is evaluated in the HallerKramer model, this will result in a model where different dissipative phenomena, both quasi-static (for frequency near zero) and dynamic (due to eddy-currents) are considered. Thus the Haller-Kramer model provides a physical basis for loss separation.

Haller and Kramer [2] based their analysis on the existence of different dissipative processes, one due to eddy currents $\left(E_{\text {eddy }}\right)$ and another due to creation and annihilation of domain walls $\left(E_{\text {wall }}\right)$, see Eqs. (1)-(2). $E_{\text {wall }}=n \gamma A$ where $A$ is domain area area, which is given by the product $A=e w$ and $\gamma$ is domain wall energy. Equations (1) and (2) are valid for frequency zero, and also for any frequency. Note that $n$ varies with the frequency $f$. Note also that it is possible to write either $n(f)$ or $n$. The first part of Eq. (1) is the magnetostatic energy $E_{m}$. For magnetostatic energy, if the number of poles (as North or South) is taken into account, then it results in $n+1$. For the second part of Eq. (1), the domain wall (or exchange energy) is taken into account, resulting in $n$ (and not $n+1$ ). Haller and Kramer [2] assumed that the system is in quasi-stationary state, see Eq. (3). The number of domain walls $n$ is function of frequency $f$, as experimentally observed [3]. The model of Haller and Kramer [2] is for only one grain with length $L$, thickness $e$, and width $w$, see Fig. $1 . \quad \beta$ is a constant found from the WSK theory [5]. For a half cycle $(f / 2), E_{\text {eddy }}$ is given by Eq. (4).

$$
\begin{aligned}
& E \cong \frac{1}{n+1} E_{m}+n \gamma A, \\
& E=1.7 M_{s}^{2}(D / L)+\gamma / D, \\
& \frac{\mathrm{d} E}{\mathrm{~d} n}=\frac{\mathrm{d}\left(E_{\text {eddy }}+E_{\text {wall }}\right)}{\mathrm{d} n}=0,
\end{aligned}
$$




$$
E_{e d d y}=\int_{0}^{f / 2} \beta n v^{2} \mathrm{~d} t .
$$

The domain wall velocity $v$ is given by Eq. (5), for sinusoidal waveform. The constant $\beta$ is given by Eq. (6) [8]. For sinusoidal waveform Eq. (4) becomes Eq. (7). $c$ is speed of the light, $\rho$ is resistivity, $B_{s}$ is the induction of saturation. $B_{s}$ is the magnetization of saturation $M_{s}$ times $4 \pi$, i.e., $B_{s}=4 \pi M_{s}$. From Eq. (3), the equilibrium distance between domain walls $D(f)$ is found, see Eq. (8).

$$
\begin{aligned}
& v=\left(\frac{L \pi f}{n}\right) \cos (2 \pi f t), \\
& \beta=1.05 \frac{16 A e B_{s}^{2}}{\rho \pi^{3} c^{2}}, \\
& E_{\text {eddy }}=\beta \frac{L^{2} \pi^{2} f}{4 n}, \\
& D(f)=\frac{L}{n(f)}=\frac{2}{\pi} \sqrt{\frac{\gamma A}{\beta f}} .
\end{aligned}
$$

The eddy current term is from the Pry and Bean model [9], taking into account the anomalous factor. Thus, the separation between classical eddy $P_{\text {clas }}$ and anomalous $P_{\text {an }}$ is artificial. The Pry and Bean result [9] is $P_{\text {clas }}+P_{\text {an }}=\xi(D / e) P_{\text {clas }}$. The Pry and Bean original calculation is for a very simple domain structure, which may be valid for grain oriented steels. For other materials, as non-oriented steels with complex domain and domain wall structure, the non-dimensional anomalous coefficient $\xi$ is defined.

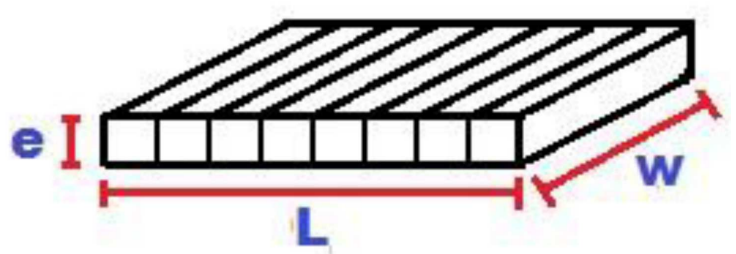

Fig. 1. Scheme of the domain structure in the HallerKramer model, with neighbor domains magnetized in opposite directions.

One of the main predictions of Eq. (8) is that $D(f)$ varies with $1 / \sqrt{ } f$. This law has been experimentally verified [3]. There are many reports [10-12], that the number of domain walls increases with frequency, following a law of type $n \sim f^{1 / 2}$. This is used in the model for anomalous losses given by Eq. (9) where $a$ is an experimental parameter [13]:

$$
P_{\text {an }}=a \frac{1}{n} L^{1 / 2} \frac{1}{\rho} e^{2} B_{\max }^{2} f^{3 / 2} .
$$

The total losses are $P_{t}=P_{h}+P_{c l}+P_{a n}$, where $P_{t}$ is the total experimental losses, and $P_{c l}$ is Eq. (10), and $P_{h}$ is Eq. (11). Expression (10) assumes perfect flux penetration (i.e., no skin effect), constant permeability, and is valid only for small frequencies, less than $400 \mathrm{~Hz}$

$$
\begin{aligned}
& P_{\mathrm{cl}}=\frac{\pi^{2}}{6} \frac{1}{\rho} e^{2} B_{\max }^{2} f^{2}, \\
& P_{h}=f \oint H \mathrm{~d} B .
\end{aligned}
$$

The frequency $f$ exponents 1, 2, and 1.5 (1st, 2nd and 3rd terms, respectively) in Eqs. (12)-(14) can be derived with the Haller-Kramer model. As the Haller-Kramer model takes into account all the energy of the system, the loss separation procedure can be obtained from it. Equation (10) is for sinusoidal waveform, but it can be adapted for other waveforms, by means of the constant $C_{e}$, see Eqs. (12)-(14). Loss separation can be applied to different waveforms, and not only for sinusoidal [14]. For example, the square waveform was considered in that previous study [14].

Alternative expressions for the total losses $P_{t}$ given by the three losses terms are Eqs. (12) and (13). $C_{h}, C_{e}$, and $C_{a}$ are experimental constants. $q$ and $m$ are nondimensional, and experimentally determined.

$$
\begin{aligned}
P_{t} & =C_{h} B^{q} f+C_{e} B^{2} f^{2}+C_{a} B^{m} f^{3 / 2}, \\
P_{t} & =C_{h} B^{(q-1)}\left(\frac{\mathrm{d} B}{\mathrm{~d} t}\right)+C_{e}\left(\frac{\mathrm{d} B}{\mathrm{~d} t}\right)^{2} \\
& +C_{a} B^{(m-3 / 2)}\left(\frac{\mathrm{d} B}{\mathrm{~d} t}\right)^{3 / 2}
\end{aligned}
$$

With proper choices of $q$ and $m$, Eq. (14) is obtained for total losses $P_{t}$ :

$$
P_{t}=C_{h}\left(\frac{\mathrm{d} B}{\mathrm{~d} t}\right)+C_{e}\left(\frac{\mathrm{d} B}{\mathrm{~d} t}\right)^{2}+C_{a}\left(\frac{\mathrm{d} B}{\mathrm{~d} t}\right)^{3 / 2} .
$$

Dlala et al. $[15,16]$ have suggested that multiple harmonics are relevant. Especially in the case of finite element models, multiple harmonics may deserve to be taken into account [17]. In this case, the total losses $P_{t}$ given by Eq. (15) can be obtained from Eq. (14). Note that Eq. (15) is a sum where the non-dimensional integer $j$ is multiplying the frequency $f . B_{j}$ is the amplitude of the $j$-th frequency harmonic of the flux density waveforms. The procedure of Dlala et al. $[15,16]$ has physical basis provided by the Haller-Kramer model

$$
P_{t}=\sum_{j=1}^{\infty}\left[C_{h} B_{j}(j f)+C_{e} B_{j}^{2}(j f)^{2}+C_{a} B_{j}^{3 / 2}(j f)^{3 / 2}\right] .
$$

\section{Loss separation and material optimization}

There are several dissipative processes inside a quasistatic hysteresis cycle: (i) domain rotation; (ii) creation and annihilation of domain walls; (iii) domain wall displacement; (iv) elimination of domain closure walls with magnetostrictive effects, see Fig. 2. The dependence with $f^{1}$ is clear, because these phenomena repeat once each hysteresis cycle, resulting in Eq. (11). 


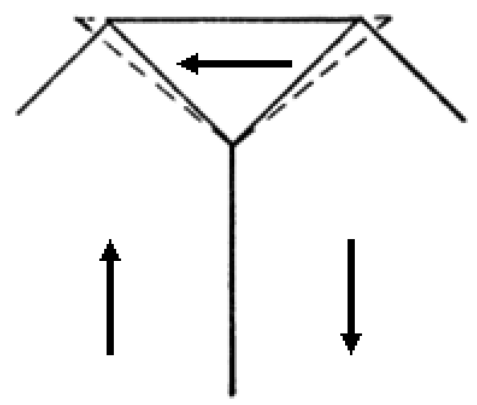

Fig. 2. Magnetostrictive effect due to formation or annihilation of $90^{\circ}$ closure domain walls. Adapted from Kittel [18].

TABLE I

Quantitative effect of several variables on the three loss components

\begin{tabular}{l|c|c|c}
\hline \hline \multicolumn{1}{c|}{ Variable } & Classical eddy & Hysteresis & Anomalous \\
\hline induction $B$ & $B_{\max }^{2}$ & $B_{\max }^{1.6-2.0}$ & $B_{\max }^{1.5-2.0}$ \\
frequency $f$ & $f^{2}$ & $f$ & $f^{3 / 2}$ \\
resistivity $\rho$ & $1 / \rho$ & - & $1 / \rho$ \\
thickness $e$ & $e^{2}$ & & $e^{2}$ \\
grain size $G_{s}$ & - & $1 / G_{s}$ & $G_{s}^{0.5}$
\end{tabular}

The dissipative mechanism presented in Fig. 2 is very relevant, for example, for grain oriented steels. The formation or annihilation of closure domain walls can generate significant noise [19]. Thus, magnetostriction can be a significant source of hysteresis losses. This means that the chemical composition of the electrical steels should be chosen in such way to have the magnetostriction constant near zero.

There is an example of soft magnetic material where domain rotation is a relevant mechanism of magnetic reversal, and this is the case of high frequency ferrites [20]. MnZn ferrites are optimized with basis on the loss separation model. Expression (11) follows $f^{1}$, whereas Eq. (10) follows $f^{2}$ and Eq. (9) follows $f^{3 / 2}$. As consequence, the strategy for material optimization in MnZn ferrites is increasing hysteresis losses Eq. (11), and decreasing anomalous losses Eq. (9), with very small grain size, less than $3 \mu \mathrm{m}$ [20], where the reversal takes place by domain rotation. For very high frequencies there is also a ferromagnetic resonance loss term, which was discussed by Van der Zaag [20]. The predictions of the loss separation model Eqs. (9)-(11) can be summarized in Tables I and II. These predictions can be very useful for designing material for high frequencies as $400 \mathrm{~Hz}$ or $1 \mathrm{kHz}$, and also for choosing the most adequate materials when designing motors that operate at variable frequencies. Vehicles with electric motors are usually designed without gearbox [21]. A common example is Tesla Model S, with a 9.73:1 gear reduction, without transmission. The motor of Tesla Model S may operate at $18000 \mathrm{rpm}$ or $300 \mathrm{~Hz}$. This shows the need of developing materials with low losses at a significant range of frequencies $(1 \mathrm{~Hz}$ to $1 \mathrm{kHz})$.
TABLE II

Qualitative effect of several variables on the three loss components

\begin{tabular}{l|c|c|c}
\hline \hline \multicolumn{1}{c|}{ Increase of the variable } & Eddy & Hysteresis & Anomalous \\
\hline $\mathrm{Si}$ & decreases & decreases & decreases \\
texture (improvement of) & - & decreases & decreases \\
number of domain walls $(n)$ & - & increases & decreases \\
plastic deformation (rolling) & - & increases & decreases \\
applied stress (compression) & - & increases & increases \\
inclusions & - & increases & (no effect)
\end{tabular}

The easiest way to get low losses in a wide frequency range is reducing thickness and increasing resistivity this will reduce the dynamical part of the losses, the classical eddy plus anomalous (see Table I).

As a remark, it should be reminded that the Prigogine minimum energy production can be used for modelling the evolution of the domain and domain wall structures as function of time. Thus, the Haller-Kramer model deserves more attention by the scientific community.

\section{Conclusions}

The Haller-Kramer model was discussed. It provides physical basis for loss separation. The Prigogine minimum entropy production principle can be applied for modelling domains and domain walls structure. The effect of several microstructural variables on losses (and mechanical strength) was discussed with basis on the loss separation procedure.

\section{Acknowledgments}

The author thanks financial support from FAPERJ (Fundação Carlos Chagas Filho de Amparo à Pesquisa do Estado do Rio de Janeiro) and CNPq (Conselho Nacional de Desenvolvimento Cientifico e Tecnológico).

\section{References}

[1] V.E. Legg, Bell Syst. Techn. J. 15, 39 (1936).

[2] T.R. Haller, J.J. Kramer, J. Appl. Phys. 41, 1034 (1970).

[3] T.R. Haller, J.J. Kramer, J. Appl. Phys. 41, 1036 (1970).

[4] I. Prigogine, Introduction to the Theory of Irreversible Processes, 3rd ed., Wiley, New York 1967, p. 83.

[5] A.T. Winfree, J. Chem. Educat. 61, 661 (1984).

[6] L.M. Brown, Dislocations in Solids, Vol. 11, Eds. F.R.N. Nabarro, M.S. Duesbery, Elsevier, 2002, p. 193.

[7] I. Prigogine, Europ. J. Operat. Res. 30, 97 (1987).

[8] H.J. Williams, W. Shockley, C. Kittel, Phys. Rev. 80, 1090 (1950).

[9] R.H. Pry, C.P. Bean, J. Appl. Phys. 29, 532 (1958).

[10] J. Sun, J. Kramer, IEEE Trans. Magn. 14, 776 (1978). 
[11] Y. Sakaki, IEEE Trans. Magn. 16, 569 (1980).

[12] Y.H. Kim, M. Yamaguchi, K.I. Arai, IEEE Trans. Magn. 28, 2781 (1992).

[13] M.F.D. Campos, J.C. Teixeira, F.J.G. Landgraf, J. Magn. Magn. Mater. 301, 94 (2006).

[14] C. Simao, M.F.D. Campos, N. Sadowski, N.J. Batistela, F.J.G. Landgraf, J. Magn. Magn. Mater. 320, e381 (2008)

[15] E. Dlala, IEEE Trans. Magn. 45, 716 (2009).

[16] E. Dlala, M. Solveson, S. Stanton, A. Arkkio, in: Proc. IEEE Int. Electric Machines and Drives Conf. (IEMDC), 2015, p. 340
[17] G.V. Pfingsten, S. Steentjes, K. Hameyer, IEEE Trans. Industr. Electron. 64, 2538 (2017).

[18] C. Kittel, Rev. Mod. Phys. 21, 541 (1949).

[19] S. Arai, in: Proc. WMM'18, Dresden (Germany), 2018, p. 74.

[20] P.J.V. der Zaag, J. Magn. Magn. Mater. 196, 315 (1999).

[21] G. Volpe, J. Goss, I. Foley, F. Marignetti, M. Popescu, D.A. Staton, in: Proc. IEEE Vehicle Power and Propulsion Conf. (VPPC), 2017, p. 1. 